\title{
Constrained Reachability and Trajectory Generation for Flat Systems *
}

\author{
Timm Faulwasser ${ }^{\mathrm{a}}$, Veit Hagenmeyer ${ }^{\mathrm{b}}$ and Rolf Findeisen ${ }^{\mathrm{c}}$ \\ ${ }^{a}$ Laboratoire d'Automatique, Ecole Polytechnique Fédérale de Lausanne, \\ Switzerland \\ b BASF SE, Ludwigshafen, Germany \\ ${ }^{\mathrm{c}}$ Institute for Automation Engineering, Otto von Guericke University Magdeburg, \\ Germany
}

\begin{abstract}
We consider the problem of trajectory generation for constrained differentially flat systems. Based on the topological properties of the set of admissible steady state values of a flat output we derive conditions which allow for an a priori verification of the feasibility of constrained set-point changes. We propose to utilize this relation to generate feasible trajectories. To this end we suggest to split the trajectory generation problem into two stages: (a) the planning of geometric reference paths in the flat output space combined with (b) an assignment of a dynamic motion to these paths. This assignment is based on a reduced optimal control problem. The unique feature of the approach is that due to the specific construction of the paths the optimal control problem to be solved is guaranteed to be feasible. To illustrate our results we consider a Van de Vusse reactor as an example.
\end{abstract}

Key words: reachability; constraints; differentially flat systems; optimal control; feasibility; path following;

\section{Introduction}

The problem of transition between set-points is an important control task. Transition problems are usually approached either by feedback control or via

^ . Corresponding author T. Faulwasser, Phone: +41 (0) 21693 7341, Fax: +41 (0) $21693 \quad 25$ 74. Email address: timm.faulwasser@epfl.ch, veit.hagenmeyer@basf.com,rolf.findeisen@ovgu.de

Preprint 
two-degree-of-freedom control schemes. While the first approach might lead to sophisticated feedback controllers with aggressive behavior, the latter combines feedforward inputs, which transfer the system smoothly from one setpoint to another one, with typically rather simple feedback structures, cf. $[4,8,11]$. Feedforward inputs which ensure nominal set-point transition can be obtained by solving an optimal control problem $[2,15]$ or by using system inversion techniques and/or flatness properties, cf. [4,7,8,10,16,25]. These approaches, however, share the general limitation that nonlinear dynamics and constraints on states and/or inputs are difficult to handle. These difficulties stem from the fact that for constrained nonlinear systems confirming whether one set-point is reachable from another one is usually achieved by computation of an admissible trajectory.

In order to combine optimal control methods with system inversion techniques we focus on the special case of differentially flat systems. Exploiting flatness in the context of dynamic optimization and trajectory generation has been considered previously, see [19-21,25-27]. Generally speaking, these works convert an infinite dimensional optimal control problem into a finite dimensional static optimization problem by describing the system evolution via parametrized functions, e.g. splines, in a flat output space. One common restriction of these works is that for nonlinear flat systems subject to input and state constraints, the existence of admissible solutions is in general not guaranteed. In the present contribution we tackle this limitation.

To this end we investigate a reachability condition for flat systems, which allows to confirm set-point reachability a priori, i.e. without explicit computation of admissible solutions. The condition is based on a relation between the constrained reachability of flat systems and the topology of the set of steady state values of a flat output. Similar observations are made but not further investigated in $[18,23]$. We propose to utilize this relation in terms of a two-stage-approach to trajectory generation: (a) the planning of a geometric reference path in a flat output space which connects the set points, and (b) assigning an admissible dynamic trajectory to this curve. The first step is subject to specific conditions and precomputed while in the second step a small dimensional optimal control problem with strict feasibility guarantee is formulated.

The remainder of the present contribution is structured as follows: In Section 2 we present the problem setting, briefly recall the property of differential flatness, and present the main reachability result. The proof of this result prepares the ground for a two-stage approach to trajectory generation for flat systems which is presented in Section 3. Section 4 considers a nonlinear Van de Vusse reactor subject to state and input constraints as an example. 


\section{Notation}

The image of a set $\mathcal{A} \subset \mathbb{R}^{n}$ under a map $f: \mathbb{R}^{n} \rightarrow \mathbb{R}^{m}$ is denoted as $f(\mathcal{A})$.

The interior of a compact set $\mathcal{B}$ is written as $\operatorname{int}(\mathcal{B})$. The $k$-th time derivative of a function $r:[0, \infty) \rightarrow \mathbb{R}$ is written as $\frac{d^{k} r(t)}{d t^{k}}$ or more conveniently $r^{(k)} . \mathcal{C}^{k}$ denotes the set of $k$-times continuously differentiable functions. The solution at time $t$ of an ODE $\dot{x}=f(x, u)$ starting at $x(0)=x_{0}$ and driven by an input $u:[0, t] \rightarrow \mathbb{R}^{m}$ is written as $x\left(t, x_{0} \mid u(\cdot)\right)$.

\section{Problem Statement and Constrained Reachability Result}

We consider nonlinear systems of the form

$$
\begin{aligned}
& \dot{x}=f(x, u), \quad x(0)=x_{0}, \\
& y=h\left(x, u, \dot{u}, \ldots, u^{(l)}\right) .
\end{aligned}
$$

The states $x \in \mathbb{R}^{n}$ and inputs $u \in \mathbb{R}^{m}$ are constrained by simply connected compact sets $\mathcal{X} \subset \mathbb{R}^{n}$ and $\mathcal{U} \subset \mathbb{R}^{m}$. The state constraint set is described as $\mathcal{X}=\left\{x \in \mathbb{R}^{n_{x}} \mid c_{i}^{x}(x) \leq 0, \quad c_{i}^{x} \in \mathcal{C}^{0}, i=1, \ldots, n_{c x}\right\}$ and the input constraint is $\mathcal{U}=\left\{u \in \mathbb{R}^{n_{u}} \mid c_{i}^{u}(u) \leq 0, \quad c_{i}^{u} \in \mathcal{C}^{0}, i=1, \ldots, n_{c u}\right\}$. The control objective is to generate constraint consistent input and state trajectories as well as a finite time $T$, such that the system is driven from one set-point $\left(x_{0}, u_{0}\right) \in \mathcal{X} \times \mathcal{U}$ to another set-point $\left(x_{T}, u_{T}\right) \in \mathcal{X} \times \mathcal{U}$, whereby

$$
0=f\left(x_{i}, u_{i}\right), \quad i \in\{0, T\}
$$

holds. Formally this can be stated as follows.

Problem 1 (Constrained set-point transition) Given system (1), an initial set-point $\left(x_{0}, u_{0}\right) \in \mathcal{X} \times \mathcal{U}$, and a target set-point $\left(x_{T}, u_{T}\right) \in \mathcal{X} \times \mathcal{U}$. Compute

(1) a finite transition time $T \in[0, \infty)$;

(2) and an admissible input signal $u:[0, T] \rightarrow \mathcal{U} \subset \mathbb{R}^{m}$ such that the system trajectory satisfies

$$
\begin{aligned}
& \forall t \in[0, T]: \quad x\left(t, x_{0} \mid u(\cdot)\right) \in \mathcal{X}, \\
& i \in\{0, T\}: \quad\left(x\left(i, x_{0} \mid u(\cdot)\right), u(i)\right)=\left(x_{i}, u_{i}\right) .
\end{aligned}
$$

Note that the solution to this problem is usually not unique. Part (2) refers to the general problem of reachability in the presence of constraints on states and inputs. Part (1) requires to transfer the system between the set-points in finite 


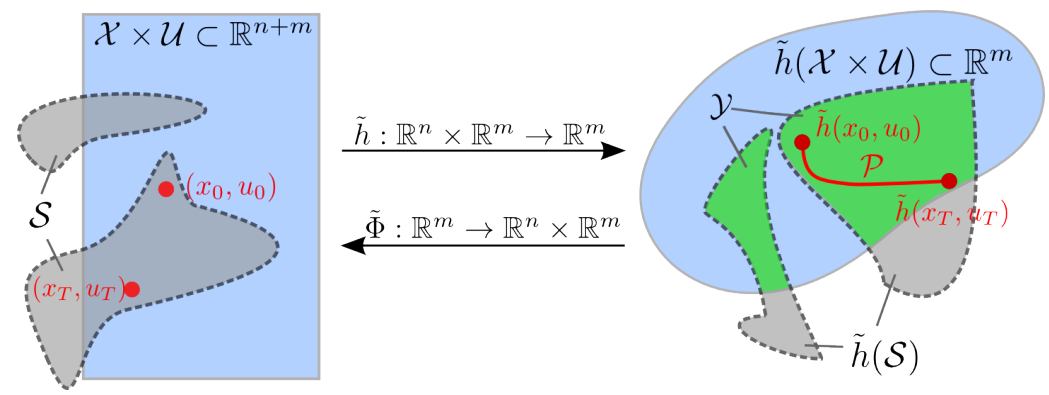

Fig. 1. Illustration of the main idea of Theorem 1.

time. Often this problem is tackled as an optimal control problem: either with an a priori choice of the transition time $T$ or formulated as a free-end-time optimal control problem, cf. $[2,15]$. However, it is in general difficult to verify a priori whether - given system (1), the constraint sets $\mathcal{X}, \mathcal{U}$ and set-points $\left(x_{i}, u_{i}\right), i \in\{0, T\}$-Problem 1 is feasible.

To provide sufficient conditions on finite-time set point reachability we restrict the further considerations to the class of differentially flat systems [7].

\section{Definition 1 (Differentially flat system)}

Consider the system (1a). If there exists a variable $\xi=\left(\xi_{1}, \ldots, \xi_{n_{\xi}}\right)^{T}$ with $\operatorname{dim} \xi=n_{\xi}=\operatorname{dim} u=m$, such that the following statements hold at least locally:

(1) The variable $\xi$ can be written as a function of the state variables $x=$ $\left(x_{1}, \ldots, x_{n}\right)^{T}$, the input variables $u=\left(u_{1}, \ldots, u_{m}\right)^{T}$ and a finite number of time derivatives of the input variables

$$
\xi=g\left(x, u_{1}, \ldots, u_{1}^{\left(l_{1}\right)}, \ldots, u_{m}, \ldots, u_{m}^{\left(l_{m}\right)}\right) .
$$

(2) The system variables $x$ and $u$ can be expressed as functions of the variable $\xi=\left[\xi_{1}, \ldots, \xi_{m}\right]^{T}$ and a finite number of time-derivatives of $\xi$. Hence there exist maps $\Phi_{1}: \mathbb{R}^{\kappa} \rightarrow \mathbb{R}^{n}, \kappa=\sum_{i=1}^{m} k_{i}$ and $\Phi_{2}: \mathbb{R}^{\kappa+m} \rightarrow \mathbb{R}^{m}$ such that

$$
\begin{aligned}
& x=\Phi_{1}\left(\xi_{1}, \ldots, \xi_{1}^{\left(k_{1}-1\right)}, \ldots, \xi_{m}, \ldots, \xi^{\left(k_{m}-1\right)}\right) \\
& u=\Phi_{2}\left(\xi_{1}, \ldots, \xi_{1}^{\left(k_{1}\right)}, \ldots, \xi_{m}, \ldots, \xi_{m}^{\left(k_{m}\right)}\right) .
\end{aligned}
$$

(3) The components of $\xi$ are differentially independent, they do not fulfill any differential equation.

Then $\xi$ is called a flat output of (1a). Furthermore, (1a) is called a (differentially) flat system.

As is well known the flatness property can be exploited in control tasks such as trajectory generation and set-point changes, see e.g. [7,16,25]. We will utilize flatness to tackle Problem 1. Thus we assume the following. 


\section{Assumption 1 (Flat system)}

System (1a) is a differentially flat system and (1b) is one of its flat outputs. Furthermore, the according maps $\Phi_{1}, \Phi_{2}$ from (4) are continuous on sufficiently large subsets $\mathcal{I} \subseteq \mathbb{R}^{\kappa}, \mathcal{J}=\mathcal{I} \times \hat{\mathcal{J}} \subseteq \mathbb{R}^{\kappa+m}$ of their domains such that

$$
\mathcal{X} \times \mathcal{U} \subseteq \Phi_{1}(\mathcal{I}) \times \Phi_{2}(\mathcal{J})
$$

holds.

In essence, this assumption states that flatness and continuity of the parametrizations (4) hold for all $(x, u) \in \mathcal{X} \times \mathcal{U}$. Relaxing this will be discussed in Remark 1.

For brevity of presentation the set

$$
\mathcal{S}:=\left\{(x, u) \in \mathbb{R}^{n} \times \mathbb{R}^{m} \mid f(x, u)=0\right\}
$$

denotes the set of steady states of (1) and the map $\tilde{h}: \mathbb{R}^{n} \times \mathbb{R}^{m} \rightarrow \mathbb{R}^{m}$

$$
\tilde{h}(x, u):=h(x, u, 0, \ldots, 0)
$$

is the output map corresponding to stationary inputs of (1). The map $\tilde{\Phi}$ : $\mathbb{R}^{m} \rightarrow \mathbb{R}^{n} \times \mathbb{R}^{m}$

$$
\tilde{\Phi}(y)=\left(\begin{array}{l}
\Phi_{1}\left(y_{1}, 0, \ldots, 0, y_{2}, 0, \ldots, y_{m}, 0 \ldots, 0\right) \\
\Phi_{2}\left(y_{1}, 0, \ldots, 0, y_{2}, 0, \ldots, y_{m}, 0 \ldots, 0\right)
\end{array}\right)
$$

is the stationary version of (4). These notions allow us to define the set of stationary outputs which are consistent with the constraints.

Definition 2 (Consistent stationary outputs) The sets $\mathcal{Y} \subseteq \hat{\mathcal{Y}} \subset \mathbb{R}^{m}$

$$
\begin{aligned}
& \hat{\mathcal{Y}}=\{y=\tilde{h}(x, u) \mid(x, u) \in \mathcal{S} \cap(\mathcal{X} \times \mathcal{U})\}, \\
& \mathcal{Y}=\{y=\tilde{h}(x, u) \mid(x, u) \in \mathcal{S} \cap \operatorname{int}(\mathcal{X} \times \mathcal{U})\}
\end{aligned}
$$

are called set of constraint consistent steady state outputs and set of strongly constraint consistent steady state outputs, respectively.

The following main reachability result sets the basis for the further considerations.

\section{Theorem 1 (Feasibility of set-point transitions)}

Given a flat system which satisfies Assumption 1. Then for any pair of setpoints $\left(x_{i}, u_{i}\right), i \in\{0, T\}$ for which there exists an open, simply connected and bounded set $\mathcal{K} \subseteq \mathcal{Y} \subset \mathbb{R}^{m}$ such that

$$
\tilde{h}\left(x_{i}, u_{i}\right) \in \mathcal{K}, \quad i \in\{0, T\}
$$


holds, the constrained finite-time set-point transition, as defined in Problem 1, is feasible.

Some comments are in order before we prove this result. The main idea behind the theorem is to split the set-point transition problem into two stages: (a) planning of paths connecting the set-points in the flat output space, and (b) assignment of an admissible dynamic reference evolution to this path. Figure 1 shows a graphical interpretation of this approach. In the path planning stage a stationary problem description is used. This means that the set-points and constraints are mapped from the extended state space-i.e. the space of $(x, u) \in \mathbb{R}^{n} \times \mathbb{R}^{m}$ - to the flat output space via the map (7). In essence condition (10) implies that in the flat output space the set-points are connectable via a smooth path $\mathcal{P}$ that is contained in one component of $\mathcal{Y}$ (dark grey color in Figure 1). Thus each point $p$ on the connecting path $\mathcal{P}$ corresponds to a strongly constraint consistent steady state, i.e., $\tilde{\Phi}(p)=(x, u) \in \operatorname{int}(\mathcal{X} \times \mathcal{U})$. In the second stage the dynamic aspects of the transition problem are considered, i.e. we show that (10) is sufficient to ensure the existence of an admissible transition trajectory which travels exactly along the path $\mathcal{P}$. The ideas behind the theorem and its proof lead to the derivation of a trajectory generation approach with feasibility guarantees.

\section{Proof of Theorem 1}

The proof is constructive and structured as follows: Based on some technical considerations, we reformulate the set-point transition problem as a pathfollowing problem in a flat output space of (1a). Specifically, we show how to assign constraint consistent motions to reference paths which connect the desired set-points. Finally, we conclude from the existence of an admissible solution to this reformulated problem that the conditions of Theorem 1 are sufficient to ensure feasibility of Problem 1.

Clearly, the conditions of Theorem 1 imply that the stationary output values that correspond to the set-points - i.e. the output values $\tilde{h}\left(x_{0}, u_{0}\right)$ and $\tilde{h}\left(x_{T}, u_{T}\right)$ - can be connected by a continuous path contained in $\mathcal{K} \subseteq \mathcal{Y}$. Since we require that $\tilde{h}\left(x_{0}, u_{0}\right)$ and $\tilde{h}\left(x_{T}, u_{T}\right)$ are contained in an open, simply connected set $\mathcal{K}$ we can also ensure the existence of connecting paths which are sufficiently often continuously differentiable.

Lemma 1 (Existence of $\mathcal{C}^{k}$ connecting paths)

Consider an open, simply connected and bounded subset $\mathcal{K}$ of $\mathbb{R}^{m}$. Any two 
points $p_{0}, p_{T} \in \mathcal{K}$ can be connected by a regular path ${ }^{1}$

$$
\mathcal{P}=\left\{y \in \mathbb{R}^{m} \mid \theta \in\left[\theta_{0}, \theta_{T}\right] \mapsto y=p(\theta)\right\} \subset \mathcal{K}
$$

with a parametrization $p:\left[\theta_{0}, \theta_{T}\right] \rightarrow \mathcal{P}, p(\theta) \in \mathcal{C}^{k}$, where $k$ is an arbitrary number $k \in \mathbb{N}$.

The proof follows directly from the fact that simple connectedness implies path connectedness and the fact that $\mathcal{K}$ is open.

\section{Definition 3 (Steady state consistent path)}

$A$ path $\mathcal{P}(11)$ is called steady state consistent with respect to (1) and the constraints $\mathcal{X}, \mathcal{U}$, if $\mathcal{P} \subseteq \hat{\mathcal{Y}}$. If $\mathcal{P} \subseteq \mathcal{Y} \subseteq \hat{\mathcal{Y}}$ holds, then we call $\mathcal{P}$ strongly steady state consistent.

Using this definition one can paraphrase the condition (10) of Theorem 1 . In essence, (10) implies that the stationary output values $\tilde{h}\left(x_{0}, u_{0}\right)$ and $\tilde{h}\left(x_{T}, u_{T}\right)$ can be connected by a strongly steady state consistent path. In order to avoid technicalities we make the following temporary assumption.

\section{Assumption 2 (Known path parametrization)}

Given two set-points $\left(x_{0}, u_{0}\right),\left(x_{T}, u_{T}\right)$, which fulfill the conditions of Theorem 1. We assume that a regular, strongly steady consistent path $\mathcal{P}(11)$ is known, such that

$$
\begin{aligned}
& \text { (1) its parametrization satisfies } p(\theta) \in \mathcal{C}^{\hat{k}} \text {, whereby } \hat{k}=\max _{i}\left\{k_{i}\right\} \text { with } k_{i}, i \in \\
& \{1, \ldots, m\} \text { from }(4 \mathrm{~b}) \text {; } \\
& \text { (2) and } \mathcal{P} \text { connects the set-points, i.e. } p\left(\theta_{i}\right)=\tilde{h}\left(x_{i}, u_{i}\right), i \in\{0, T\} .{ }^{2}
\end{aligned}
$$

Reformulation as Path-following Problem

Instead of solving the set-point transition problem directly, we reformulate Problem 1 as a constrained path-following problem. This means that we consider the problem of steering the system (1a) from one set-point to another along the path $\mathcal{P}$ from Assumption 2. More formally, this problem is stated as follows:

\section{Problem 2 (Constrained exact path following)}

Given the constrained system (1), and a path $\mathcal{P}(11)$ to be followed. Compute an input signal $u:[0, T] \rightarrow \mathcal{U}$ and a transition time $T \in[0, \infty)$ such that the following conditions are satisfied:

1 A path is called regular when its parametrization is locally bijective [28].

2 We will comment on the computation of paths fulfilling this assumption in Section 3 . 

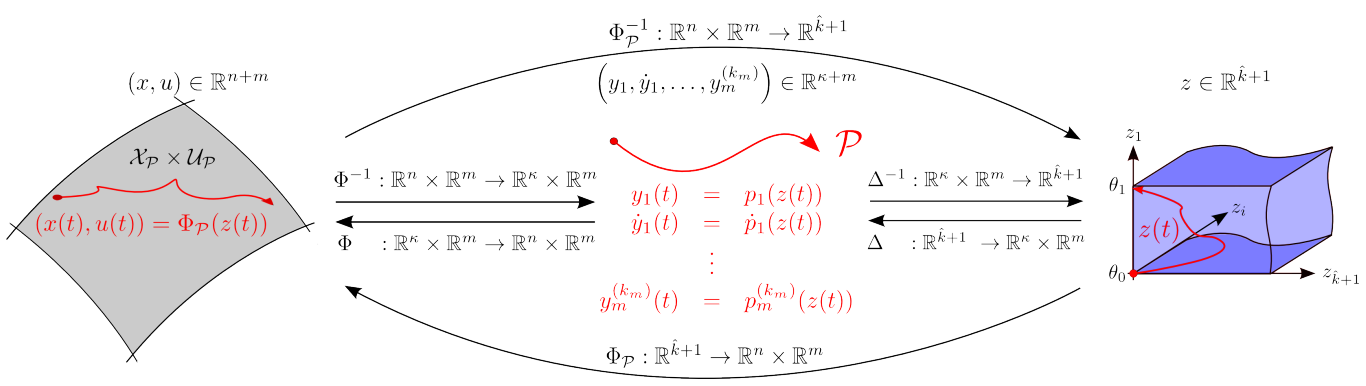

Fig. 2. Illustration of the main idea of Theorem 2.

(1) Exact Path Following: The system output (1b) moves from a consistent initial condition $\left.h(\cdot)\right|_{t=0}=p\left(\theta_{0}\right)$ in forward direction exactly along the path $\mathcal{P}$ to $\left.h(\cdot)\right|_{t=T}=p\left(\theta_{T}\right)$.

(2) Constraint Satisfaction: The feedforward input $u(\cdot)$ satisfies the input constraints and the corresponding system trajectory satisfies the state constraints, i.e. for all $t \in[0, T]: u(t) \in \mathcal{U}$ and $x\left(t, x_{0} \mid u(\cdot)\right) \in \mathcal{X}$.

At first glance it seems as if the introduction of the path constraint - part 1 of Problem 2-complicates the set-point transition problem. Essentially, the formulation of Problem 2 implies an $m$-dimensional algebraic constraint

$$
h\left(x, u, \dot{u}, \ldots, u^{(l)}\right)-p(\theta(t))=0
$$

for the system. The motivation to reformulate the set-point transition (Problem 1) as a path-following problem (Problem 2) is as follows: if we can show feasibility of the latter problem for paths contained in $\mathcal{Y}$ then the existence of an admissible solution to Problem 1 follows directly via Assumption 2 and Lemma 1.

The key point of path-following problems is that the timing $t \mapsto \theta(t)$ to move along the path is not specified a priori, rather it is an additional degree of freedom during the controller design, cf. $[1,6]$. We use this freedom of choosing $t \mapsto \theta(t)$ to rigorously ensure the satisfaction of state and input constraints. ${ }^{3}$ Without loss of generality we restrict ourselves to forward movement along the path, i.e. $\dot{\theta} \geq 0$ should hold for all $t \in[0, T]$.

Next we show that flatness of (1a) enables to state sufficient conditions for exact path following in the presence of constraints. As a preliminary step we recall sufficient conditions for unconstrained exact path following of flat systems.

\section{Lemma 2 (Unconstrained exact path following)}

3 The idea to treat the timing of the reference as a degree of freedom is related to the concept of orbital flatness that relies on the use of a time-rescaling to identify a flat output $[9,22,30]$. A detailed investigation of the relation between orbital flatness and path-following is, however, beyond the scope of this work. 
Given an unconstrained nonlinear flat system (1a) and any regular path $\mathcal{P}$ specified by an a priori known parametrization $p(\theta)$ in a flat output space $(1 \mathrm{~b})$.

Furthermore, suppose that

(i) the parametrization $p(\theta) \in \mathcal{C}^{\hat{k}}$, where $\hat{k}=\max \left\{k_{i}\right\}$ and the constants $k_{i}, i \in\{1, \ldots, m\}$ are defined by $(4 \mathrm{~b})$;

(ii) the initial condition $x_{0}$ of $(1 \mathrm{a})$ is consistent with $\mathcal{P}$ and $\theta(t) \in \mathcal{C}^{\hat{k}}$, i.e.

$$
x_{0}=\left.\Phi_{1}\left(p_{1}(\theta(t)), \ldots, \frac{d^{k_{m}-1} p_{m}}{d t^{k_{m}-1}}\right)\right|_{t=0}
$$

where $\dot{\theta}(t) \geq 0$ and $\theta(0)=\theta_{0}$ and $\theta(T)=\theta_{T}$.

Then the feedforward input

$$
u_{\mathcal{P}}(t) \quad=\quad \Phi_{2}\left(p_{1}(\theta(t)), \ldots, \frac{d^{k_{1}} p_{1}}{d t^{k_{1}}}, \ldots, \frac{d^{k_{m}} p_{m}}{d t^{k_{m}}}\right)
$$

guarantees that the system (1a), starting from $x_{0}$, follows the path $\mathcal{P}$ exactly in forward direction and reaches $p\left(\theta_{T}\right)$ at $t=T$.

The proof of this lemma follows directly by calculation of the time derivatives of $p(\theta(t))$, use of $(4 \mathrm{~b})$ and the ideas presented in $[11,24]$.

The challenging part is to extend Lemma 2 such that constraints on inputs and states can be considered. To achieve such an extension we use a key observation from Lemma 2: due to its flatness, system (1a) under the feedforward control (14) can be described via the timing $\theta(t) \in \mathcal{C}^{\hat{k}}$. This means that the timing along the path implicitly defines the states and inputs of (1a):

Theorem 2 (Equivalence to single input system) Given a nonlinear flat system (1), and a regular path $\mathcal{P}$ specified by an a priori known parametrization $p(\theta)$ in a flat output space of the system. Suppose that the conditions of Lemma 2 are satisfied. Then the dynamics of the nonlinear MIMO system (1) under the feedforward control $u_{\mathcal{P}}(\cdot)$ from $(14)$, where $\theta(t)$ is of class $\mathcal{C}^{\hat{k}}$, are equivalent to a linear, single input system in Brunovský normal form

$$
\begin{array}{ccc}
\dot{z}_{1} & = & z_{2} \\
\dot{z}_{2} & = & z_{3} \\
\vdots & & \vdots \\
\dot{z}_{\hat{k}+1}= & \theta^{(\hat{k}+1)}(t) .
\end{array}
$$

Proof. The main idea of the theorem is sketched in Figure 2. We show that the application of (14) to system (1) guarantees that states and inputs of (1) 
can be uniquely calculated from the state of the single input system (15) and vice versa. ${ }^{4}$ Flatness of system (1a) implies that the feedforward-controlled system is equivalent to a MIMO system in Brunovský normal form [11]. If the system (1a) is controlled via the input (14) we know from Lemma 2 that the flat output $(1 \mathrm{~b})$ has to be on the path $\mathcal{P}$. This implies that the flat output and its time-derivatives have to satisfy

$$
\forall i \in\{1, \ldots, m\}, \forall j \in\left\{0, \ldots, k_{m}\right\}: \quad y_{i}^{(j)}(t)=p_{i}^{(j)}(\theta(t))
$$

Consider the substitution

$$
z:=\left(z_{1}, z_{2}, \ldots, z_{\hat{k}+1}\right)^{T}=\left(\theta, \dot{\theta}, \ldots, \theta^{(\hat{k})}\right)^{T}
$$

Combining this with the flat parametrizations (4) yields that the states and inputs of (1) subject to the path-following input (14) can be written as

$$
\begin{aligned}
& x_{\mathcal{P}}=\Phi_{1, \mathcal{P}}\left(\theta, \dot{\theta}, \ldots, \theta^{(\hat{k}-1)}\right)=: \Phi_{1, \mathcal{P}}(z) \\
& u_{\mathcal{P}}=\Phi_{2, \mathcal{P}}\left(\theta, \dot{\theta}, \ldots, \theta^{(\hat{k})}\right)=: \Phi_{2, \mathcal{P}}(z)
\end{aligned}
$$

Whereby the map $\Phi_{\mathcal{P}}: \mathbb{R}^{\hat{k}+1} \rightarrow \mathbb{R}^{n+m}$ is the composition $(x, u)=\Phi_{\mathcal{P}}(z)=$ $\Phi \circ \Delta(z)$, cf. Figure 2 , and $\Delta: \mathbb{R}^{\hat{k}+1} \rightarrow \mathbb{R}^{\kappa+m}$ is given by

$$
\Delta: z(t) \mapsto\left(p_{1}\left(z_{1}(t)\right), \dot{p}_{1}\left(z_{1}(t)\right), \ldots, p_{m}^{\left(k_{m}+1\right)}\left(z_{1}(t)\right)\right)
$$

Provided that $\mathcal{P}$ is regular and $w$ is contained in the image of $\Delta$ we can deduce from Lemma 4, which is given in Appendix A, that $w=\Delta(z)$ is locally invertible. The output map (1b) is sufficiently often continuously differentiable and thus the inverse of $(x, u)=\Phi_{\mathcal{P}}(z)$ exists and is $z=\Phi_{\mathcal{P}}^{-1}(x, u)=\Delta^{-1} \circ$ $\Phi^{-1}(x, u)$. Taking into account that one is free to choose the highest timederivative of $\theta(t)$, i.e. for any choice of $\theta^{\hat{k}+1}(t)$, satisfying $\dot{\theta}(t) \geq 0$ and $\theta(0)=\theta_{0}$ and $\theta(T)=\theta_{T}$, system (1) subject to (14) follows the path and the map $\Phi_{\mathcal{P}}: \mathbb{R}^{\hat{k}+1} \rightarrow \mathbb{R}^{n+m}$ is invertible. W.l.o.g. regard $\theta^{\hat{k}+1}(t)=v(t)$ as virtual input. It follows that system (1) subject to the feedforward control (14) is equivalent to (15).

Subsequently, we use (16) and rewrite (15) briefly as $\dot{z}=A z+B v$, whereby the matrices $A \in \mathbb{R}^{(\hat{k}+1) \times(\hat{k}+1)}$ and $B \in \mathbb{R}^{\hat{k}+1}$ have the obvious structure of an integrator chain. Now we can paraphrase the question for admissible solutions to Problem 2 in terms of feasibility of the following optimal control problem

$\overline{4}$ With respect to Figure 2 we can also interpret condition (13) of Lemma 2: The initial condition $x_{0}$ has to lie on the manifold of solutions of (1) that travel exactly along the path $\mathcal{P}$. In Figure 2 this manifold is denoted as $\mathcal{X}_{\mathcal{P}}$. 
(OCP):

$$
\underset{v(\cdot), T}{\operatorname{minimize}} T
$$

subject to the dynamics

$$
\dot{z}=A z+B v,
$$

the convex constraints

$$
\begin{aligned}
z(0) & =\left[\theta_{0}, 0, \ldots, 0\right]^{T} \\
z(T) & =\left[\theta_{T}, 0, \ldots, 0\right]^{T} \\
\forall t \in[0, T]: \quad z_{2}(t) & \geq 0 \\
v(t) & \in \mathcal{V} \subset \mathbb{R}
\end{aligned}
$$

and the usually nonconvex constraints

$$
\begin{array}{ll}
\forall t \in[0, T]: & x_{\mathcal{P}}(t)=\Phi_{1, \mathcal{P}}(z(t)) \in \mathcal{X} \\
& u_{\mathcal{P}}(t)=\Phi_{2, \mathcal{P}}(z(t)) \in \mathcal{U} .
\end{array}
$$

Note that this optimal control problem has a small number of linearly coupled dynamic states. The scalar measurable input function $v:[0, T] \rightarrow \mathcal{V}$ and the free end time $T$ are decision variables. And $\mathcal{V} \subset \mathbb{R}$ is an arbitrarily large convex and compact set. The constraints (18c) and (18d) enforce rest-to-rest motion from the initial path point $p\left(\theta_{0}\right)$ to the final path point $p\left(\theta_{T}\right)$. Equation (18e) implies forward movement along the path (part (1) of Problem 2). The constraints on states and inputs of the flat system (1a) are considered via the usually nonconvex description $(18 \mathrm{~g}, \mathrm{~h})$, cf. part (2) of Problem 2 . Note that the optimal solution $v^{\star}(\cdot)$ is the virtual input to the system (18b). Finally, the input applied to the nonlinear differentially flat system (1a) can be obtained from the optimal evolution $z^{\star}\left(t, z_{0} \mid v^{\star}(\cdot)\right)$ via the input parametrization (17b).

Note that-provided the system initial condition $x_{0} \in \mathcal{X}$ satisfies (13) - any admissible solution $v(\cdot)$ to (18) and the corresponding evolution of $z\left(t, z_{0} \mid v(\cdot)\right)$ describe a constraint consistent motion of the system along $\mathcal{P}$. Consequently, we can recast the question of whether there exist admissible solutions to Problem 2 as a question of sufficient feasibility conditions for the OCP (18). The next lemma summarizes such conditions.

\section{Lemma 3 (Feasibility of OCP (18))}

Suppose that Assumptions 1832 hold, then the OCP (18) has a (not necessarily unique) optimal solution with a finite minimal transition time $T^{\star}$.

The proof of this lemma is provided in Appendix B. Using the Lemma 3 we are able to state the following sufficient conditions which ensure feasibility of the exact path-following problem. 


\section{Theorem 3 (Constrained exact path following)}

Suppose that Assumptions $1 \& \mathcal{E} 2$ hold, and the initial condition $x_{0}$ of (1a) satisfies $x_{0}=\Phi_{1, \mathcal{P}}\left(z_{0}\right)$, then Problem 2 admits feasible solutions.

Proof. Assumptions $1 \& 2$ ensure that we can apply Lemma 3. Hence we know that the OCP (18) is feasible. Therefore the corresponding input and state trajectories of system (1), obtained via (17), satisfy the constraints.

The condition $x_{0}=\Phi_{1, \mathcal{P}}\left(z_{0}\right)$ states that the initial condition $x_{0}$ of (1a) is consistent with path $\mathcal{P}$, cf. condition (2) of Lemma 2 . Thus by application of Lemma 2 we conclude that system (1) can exactly follow path $\mathcal{P}$ and reaches its endpoint in finite time while the constraints on states and inputs are satisfied.

\section{Remark 1 (Continuity of flat parametrizations)}

Assumption 1 can be relaxed with respect to the continuity of the flat parametrizations $\Phi_{1}$ and $\Phi_{2}$ from (4). The proofs of Lemma 3 and Theorem 3 can be performed in similar ways if merely local continuity of (4) is assumed. To this end consider the set of stationary values of $z$ corresponding to points on the path

$$
\mathcal{Z}_{0}=\left[\theta_{0}, \theta_{T}\right] \times 0 \times \cdots \times 0 \subset \mathbb{R}^{\hat{k}+1} .
$$

To obtain the statements of Lemma 3 and Theorem 3 it suffices to suppose that $\Phi_{1, \mathcal{P}}$ and $\Phi_{2, \mathcal{P}}$ from (17) are continuous in a neighborhood of $\mathcal{Z}_{0}$.

Summarizing, we have shown that the conditions of Theorem 1 ensure the existence of paths connecting the set-points in a flat output space. Furthermore, Problem 1 can be reformulated as a path-following problem whose feasibility can be easily verified. Hence, we can conclude that the conditions of Theorem 1 ensure set-point reachability in the presence of constraints. Up to now we have assumed that a sufficiently often continuously differentiable path parametrization is available. In the next section, we show that under suitable assumptions it is possible to compute such parametrizations.

\section{Two-stage Constrained Trajectory Generation}

Subsequently, we use the OCP (18) to compute admissible solutions to setpoint transition problems. We propose to compute trajectories and suitable feedforward inputs as follows:

\section{Algorithm 1 (Two-stage trajectory generation)}

(0) Compute a (convex) inner approximation $\tilde{\mathcal{Y}}$ of $\mathcal{Y}$ such that $\tilde{\mathcal{Y}} \subseteq \mathcal{Y} \subseteq \hat{\mathcal{Y}}$. 
(1) Given two set-points $\left(x_{0}, u_{0}\right),\left(x_{T}, u_{T}\right) \in \operatorname{int}(\mathcal{X} \times \mathcal{U})$ compute a path $\mathcal{P}$ and its path parametrization $p(\theta) \in \mathcal{C}^{\hat{k}}$ which connects $\tilde{h}\left(x_{0}, u_{0}\right)$ and $\tilde{h}\left(x_{T}, u_{T}\right)$ through $\mathcal{Y}$.

(2) Compute a transition time $T<\infty$, an admissible solution $v:[0, T] \rightarrow \mathcal{V}$ to the projected $O C P(18)$, and the corresponding trajectory $z\left(t, z_{0} \mid v(\cdot)\right)$ of the virtual states $z$. Obtain the admissible feedforward input $u_{\mathcal{P}}$ : $[0, T] \rightarrow \mathcal{U}$ and the corresponding admissible trajectory $x_{\mathcal{P}}\left(t, x_{0} \mid u_{\mathcal{P}}(\cdot)\right)$ of the original system (1a) via the maps (17).

The main idea is to split the computation of solutions to the constrained trajectory generation problem (Problem 1) into an offline pre-computation (step 0) and two online stages: a path planning stage (step 1) and a dynamic assignment of constraint consistent trajectories (step 2). Note that the purpose of this algorithm is not to compute true minimum-time trajectories between considered set-points. The intention is rather to state an algorithm with strict feasibility guarantees for a class of set-points.

Step 0 refers to an offline pre-computation of the set of constraint consistent stationary values of the flat output (1b). One computes an inner approximation of the set $\mathcal{Y}$ from (13). To clarify this it is helpful to recall that flatness allows to describe the set of (unconstrained) set-points of (1a) via the map $\tilde{\Phi}: \mathbb{R}^{m} \rightarrow \mathbb{R}^{n} \times \mathbb{R}^{m}$ from (8). Clearly, the steady states (or set-points) of (1a) can be obtained as $(x, u)=\tilde{\Phi}(y)$, cf. Figure 1 . Thus the pre-computation in step (0) refers to the approximation of the preimage

$$
\mathcal{Y}=\left\{y \in \mathbb{R}^{m} \mid \tilde{\Phi}(y) \in \operatorname{int}(\mathcal{X} \times \mathcal{U})\right\}
$$

by a set $\tilde{\mathcal{Y}} \subseteq \mathcal{Y}$. In general the approximation of preimages of maps is a challenging task. To this end one can, for example, apply gridding techniques, or use methods based on interval arithmetics [13]. Note that merely the preimage of the static flat parametrizations (8) has to be approximated. Compared to the computation of the preimage of the dynamic flat parametrizations (4)i.e. the parametrization where not only the components of the flat output but also their time derivatives appear - this is easier since the domain $\mathbb{R}^{m}$ of the map $\tilde{\Phi}$ is less dimensional compared to the domains $\mathbb{R}^{\kappa}$ and $\mathbb{R}^{\kappa+m}$ of $\Phi_{1}$, respectively, $\Phi_{2}$ from (4).

In step 1 one has to compute a path connecting $\tilde{h}\left(x_{0}, u_{0}\right) \in \mathbb{R}^{m}$ with $\tilde{h}\left(x_{T}, u_{T}\right) \in$ $\mathbb{R}^{m}$ which fulfills Assumption 2. This means that the path has to lie entirely in the inner approximation $\tilde{\mathcal{Y}}$, and has to be parametrized by $p(\theta) \in \mathcal{C}^{\hat{k}}$. If one can obtain a convex inner approximation in step 0 the online path planning is significantly simplified. It suffices to compute straight lines. If a convex inner approximation cannot be obtained, or is very conservative, one can still rely on standard path-planning algorithms, cf. [14]. In step 2 it remains to compute a feasible, not necessarily optimal, solution to OCP (18). To this end 
one either solves this dynamic optimization problem directly, e.g. via multiple shooting. ${ }^{5}$

Alternatively, one can exploit the fact that the path parameter dynamics (18b) are a flat system. This allows to convert (18) directly into an finite dimensional optimization problem, cf. [19-21,25-27]. Finally, the feedforward input to (1) is obtained by mapping the solution trajectory via (17).

One might argue that the price to pay for the guaranteed feasibility are conservative solutions due to the restriction of the flat output to the path. To avoid overly restrictive solutions one can as well think about embedding parametrized surfaces with a dimension larger than one in the flat output space. This way one could reduce the conservatism of the proposed approach without loosing the feasibility guarantees. A detailed investigation on this issue is, however, beyond the scope of this work.

\section{Example: Trajectory Generation for a CSTR}

We consider a Van de Vusse reactor described by the dynamics

$$
\begin{aligned}
\dot{c}_{A} & =r_{A}\left(c_{A}, \vartheta\right)+\left(c_{I n}-c_{A}\right) u_{1} \\
\dot{c}_{B} & =r_{B}\left(c_{A}, c_{B}, \vartheta\right)-c_{B} u_{1} \\
\dot{\vartheta} & =h\left(c_{A}, c_{B}, \vartheta\right)+\alpha\left(u_{2}-\vartheta\right)+\left(\vartheta_{I n}-\vartheta\right) u_{1},
\end{aligned}
$$

where

$$
\begin{aligned}
r_{A} & =-k_{1}(\vartheta) c_{A}-k_{2}(\vartheta) c_{A}^{2}, \quad r_{B}=k_{1}(\vartheta)\left(c_{A}-c_{B}\right) \\
h & =-\delta\left(k_{1}(\vartheta)\left(c_{A} H_{A B}+c_{B} H_{B C}\right)+k_{2}(\vartheta) c_{A}^{2} H_{A D}\right) \\
k_{i}(\vartheta) & =k_{i 0} e^{\frac{-E_{i}}{\vartheta+\vartheta_{0}}}, \quad i=1,2,
\end{aligned}
$$

see also [23]. The system states $c_{A}$ and $c_{B}$, both in mol/l, refer to the concentration of substances $A$ and $B$ in the reactor, $\vartheta$ in ${ }^{\circ} \mathrm{C}$ is the reactor temperature. The inputs $u_{1}, u_{2}$ are the normalized flow rate through the reactor in $1 / \mathrm{h}$ and the temperature in the cooling jacket in ${ }^{\circ} \mathrm{C}$. The states and inputs are subject

5 One should note that via a suitable time transformation the free end-time problem (18) can be reformulated as fixed end-time problem that can be solved efficiently. Details on this can be found in $[3,17,29]$. 


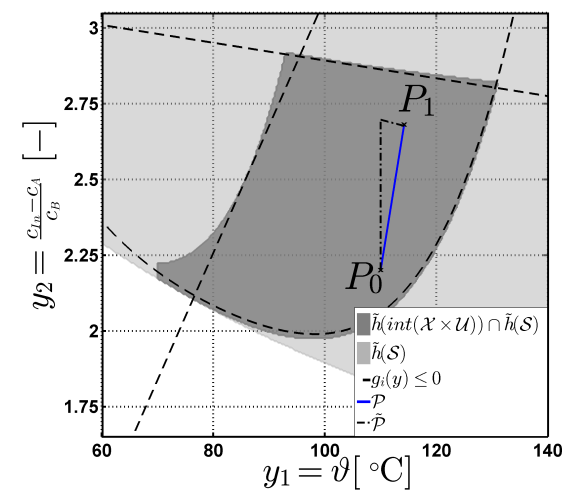

Fig. 3. Inner approximation of $\mathcal{Y}=\tilde{h}(\operatorname{int}(\mathcal{X} \times \mathcal{U})) \cap \tilde{h}(\mathcal{S})$.

to the constraints

$$
\begin{aligned}
c_{A} & \in[2.0,4.0] \frac{\mathrm{mol}}{\mathrm{l}} \quad c_{B} \in[0.1,1.2] \frac{\mathrm{mol}}{\mathrm{l}} \\
\vartheta & \in[70,200]^{\circ} \mathrm{C} \\
u_{1} & \in[3.0,35.0] \frac{1}{\mathrm{~h}} \quad u_{2} \in[10.0,150.0]^{\circ} \mathrm{C} .
\end{aligned}
$$

The system parameters can be found in [23]. There it is also shown that

$$
y=\left(\vartheta,\left(c_{I n}-c_{A}\right) / c_{B}\right)^{T}
$$

is a flat output of (21). ${ }^{6}$ To avoid long and vast expressions we give here only functional dependencies of the flat parametrizations of states and inputs

$$
\begin{aligned}
& c_{B}=\Phi_{1,1}\left(y_{1}, y_{2}, \dot{y}_{2}\right), \quad c_{A}=\Phi_{1,2}\left(y_{1}, y_{2}, \dot{y}_{2}\right) \\
& \vartheta \quad=y_{1}, \quad u_{1,2}=\Phi_{2}\left(y_{1}, y_{2}, \dot{y}_{1}, \dot{y}_{2}, \ddot{y}_{2}\right) .
\end{aligned}
$$

A detailed derivation of these maps can be found in [23].

To obtain an inner approximation $\tilde{\mathcal{Y}}$ of $\mathcal{Y}$ from $(20)$ a subset of the flat output space $(23)$ - the box $[50,150] \times[1.65,3.2]$ - is gridded with 500 sample points along each dimension. These samples are mapped via (8), which is the stationary version of (24), to the extended state space $\left(\mathbb{R}^{n} \times \mathbb{R}^{m}\right)$ to check two conditions: (a) whether a point $\left(y_{1}, y_{2}\right)$ corresponds to a physically reasonable steady state, i.e. $(x, u)=\tilde{\Phi}\left(y_{1}, y_{2}\right) \in \mathcal{S}$, and (b) to verify whether $(x, u)=\tilde{\Phi}\left(y_{1}, y_{2}\right) \in \operatorname{int}(\mathcal{X} \times \mathcal{U})$. The resulting regions are shown in Figure 3. The light grey area illustrates the image of the set of steady states in the flat output space, i.e. $\tilde{h}(\mathcal{S})$. The dark grey region depicts the intersection between $\tilde{h}(\operatorname{int}(\mathcal{X} \times \mathcal{U}))$ and $\tilde{h}(\mathcal{S})$. To get hold of the convex inner approximation $\tilde{\mathcal{Y}}$ the

6 Actually, in [23] an extended model is considered where the dynamics of the cooling jacket are described via an additional first-order linear ODE. However, one can use exactly the same steps as in [23] to show that (23) is a flat output of (21). 

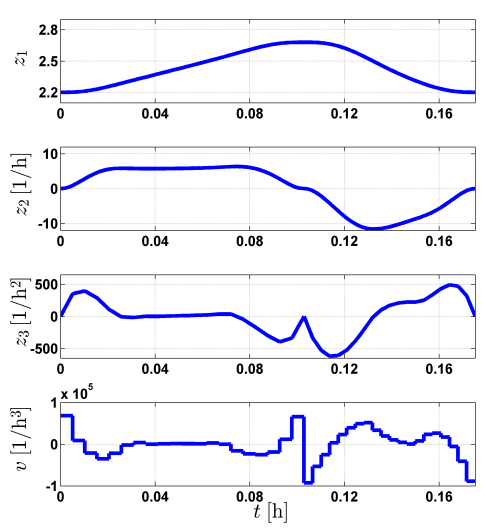

(a) Solution to $z$-dynamics via (b) Solution to (21) via OCP OCP (18).

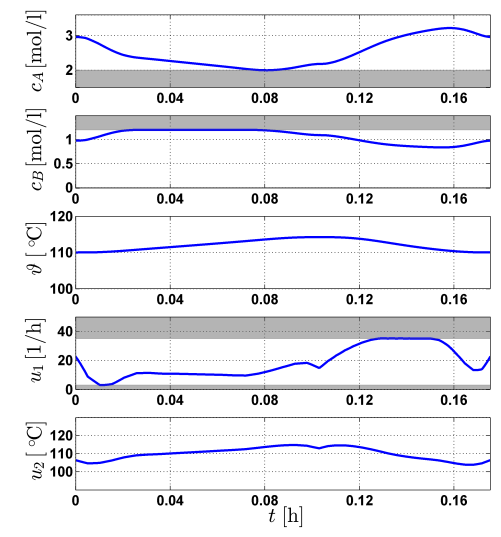

(18).

Fig. 4. Simulation results for Van de Vusse reactor.

boundary of the grey region is approximated by three polynomials $g_{i}: \mathbb{R}^{2} \rightarrow \mathbb{R}$ which are plotted as dashed curves in Figure 3.

\section{Two-stage Trajectory Generation}

In order to exemplify the computation of admissible input and state trajectories we consider two set-point changes: first connecting

$$
\begin{aligned}
x_{0} & =\left(2.95 \frac{\mathrm{mol}}{\mathrm{l}}, 0.98 \frac{\mathrm{mol}}{\mathrm{l}}, 110.00{ }^{\circ} \mathrm{C}\right) \\
u_{0} & =\left(22.7 \frac{1}{\mathrm{~h}}, 106.5{ }^{\circ} \mathrm{C}\right) \\
x_{T} & =\left(2.2 \frac{\mathrm{mol}}{\mathrm{l}}, 1.1 \frac{\mathrm{mol}}{\mathrm{l}}, 114.2{ }^{\circ} \mathrm{C}\right) \\
u_{T} & =\left(14.7 \frac{1}{\mathrm{~h}}, 113.0^{\circ} \mathrm{C}\right)
\end{aligned}
$$

and second the backward direction from $\left(x_{T}, u_{T}\right)$ to $\left(x_{0}, u_{0}\right)$. In the flat output space $(23)$ this corresponds to the transition from $P_{0}=\left(110 \mathrm{C}^{\circ}, 2.2\right)$ to $P_{1}=\left(114.21 \mathrm{C}^{\circ}, 2.68\right)$ and vice versa. The forward part from $P_{0}$ to $P_{1}$ is also considered in [23], where an intentionally slow transition along two path segments is used to generate reference and input trajectories. The path segments considered in [23] are shown as black dash-dot lines in Figure 3. The solution proposed in [23] takes 1h. Here we use the methods derived in Section 2 \& 3 to compute fast transition trajectories. The reference trajectories are obtained via the solution to the projected OCP (18). We compute the transitions such that the flat outputs (23) are restricted to the straight line connecting $P_{0}$ and $P_{1}$. This situation is also depicted in Figure 3 .

The considered path parameter dynamics (18b) are an integrator chain of length three, since the highest derivative appearing in the (dynamic) flat input 
parametrization is $\ddot{y}_{2}$ in $u_{2}=\Phi_{2,2}\left(y_{1}, \dot{y}_{1}, y_{2}, \dot{y}_{2}, \ddot{y}_{2}\right)$. We solve OCP (18) under consideration of the constraints of the original CSTR model (22) using a direct multiple shooting implementation [12].

The solutions are presented in Figure 4. Part (a) of this figures shows from top to bottom the obtained trajectories of the states $z_{1}-z_{3}$ of $(18 \mathrm{~b})$ and the corresponding virtual input $v:[0, T] \rightarrow \mathbb{R}$, which is computed as a scalar, piecewise constant function. As one can see the forward transition from $P_{0}$ to $P_{1}$ is performed in $\approx 0.11 \mathrm{~h}$. The backward transition from $P_{1}$ to $P_{0}$ is performed faster in $\approx 0.07 \mathrm{~h}$. The state and input trajectories of the CSTR (21) which correspond to this solution of the $z$-dynamics through (17) are plotted in part (b) of Figure 4. Comparing the optimal virtual input $v$ from part (a) it is clear that the small inputs for $0.02<t<0.07$ correspond to the active constraints for $u_{1}, c_{B}$ and $c_{A}$ in that time span. One should also note that the computed inputs of the real system- $u_{1}(t), u_{2}(t)$ - are rather smooth without aggressive bang-bang behavior. One reason for this is that the decision variable $v:[0, T] \rightarrow \mathcal{V}$ acts on $\theta^{(3)}(t)=v(t)$ while the highest time-derivative of $\theta(t)$ appearing in (24) is of order 2. Thus aggressive solutions in terms of the virtual input $v(t)$ are softened due to the integration $\theta^{(3)}(t)=v(t)$.

\section{Discussion \& Conclusions}

In the present contribution we investigate constrained reachability of differentially flat systems. By combining our findings with path-following concepts we can give finite-time reachability guarantees. Based on this we show how to compute admissible reference trajectories and inputs via a small dimensional optimal control problem with guaranteed feasibility. Finally, this leads to a two-stage approach to trajectory generation for constrained differentially flat systems.

\section{Acknowledgements}

The authors gratefully acknowledge the helpful discussions with Friedrich von Haeseler.

\section{A Technical Lemma}

\section{Lemma 4}

Given a regular path $\mathcal{P} \subset \mathbb{R}^{m}$ from (11) and its $k$-times continuously differ- 
entiable parametrization $\theta \mapsto p(\theta)$. Suppose that the time evolution $t \mapsto \theta(t)$ is also $k$-times continuously differentiable.

Then the map $\tilde{\Delta}: \mathbb{R}^{k+1} \rightarrow \mathbb{R}^{m \times(k+1)}$

$$
\tilde{\Delta}:\left(\theta, \dot{\theta}, \ldots, \theta^{(k)}\right) \mapsto\left(p, \dot{p}, \ldots, p^{(k)}\right)
$$

given by $p(\theta(t))$ and its time derivatives is locally invertible on open sets for all values of $\left(p, \dot{p}, \ldots, p^{k}\right)^{T}$ which are contained in the image of $\tilde{\Delta}$.

The proof of this lemma is given in $[5,6]$.

\section{B Proof of Lemma 3}

First, we will show that Assumptions $1 \& 2$ ensure the existence of feasible solutions to the OCP (18). Second, we draw upon a classical result on the existence of solutions to optimal control problems to establish the statement of Lemma 3, cf. [15].

Step 1: Consider the composition of maps $\tilde{\Phi} \circ p$, whereby $\tilde{\Phi}$ is from (8) and $p$ is the path parametrization (11). Note that $\tilde{\Phi} \circ p(\theta)=\left(\Phi_{1, \mathcal{P}}(\theta, 0, \ldots, 0), \Phi_{2, \mathcal{P}}(\theta, 0, \ldots, 0)\right)^{T}$. Assumptions $1 \& 2$ ensure that $\tilde{\Phi} \circ p:\left[\theta_{0}, \theta_{T}\right] \rightarrow \mathbb{R}^{n} \times \mathbb{R}^{m}$ is continuous. Furthermore, $\tilde{\Phi} \circ p$ maps the output path $\mathcal{P}$ to a continuous path $\mathcal{T}$

$$
\mathcal{T} \quad=\quad\left\{(x, u)^{T} \mid \theta \in\left[\theta_{0}, \theta_{T}\right] \mapsto(x, u)^{T}=\tilde{\Phi}(p(\theta))\right\}
$$

in the extended space $\mathbb{R}^{n} \times \mathbb{R}^{m}$ such that each point on $\mathcal{T}$ corresponds to a steady state. Additionally, Assumption 2 ensures that $\mathcal{T} \subset \operatorname{int}(\mathcal{X} \times \mathcal{U})$, cf. Figure 1. Thus there exists a tubular $\epsilon$-neighborhood of $\mathcal{T}$-denoted by $\mathcal{N}$ such that $\mathcal{T} \subset \mathcal{N} \subset \operatorname{int}(\mathcal{X} \times \mathcal{U})$. Due to the continuity of $\Phi_{1,2, \mathcal{P}}$ we can find trajectories $\bar{z}(t)=\left(\bar{\theta}(t), \dot{\bar{\theta}}(t), \ldots, \bar{\theta}^{(\hat{k})}(t)\right)^{T}$ such that $\bar{z}(0)=\left(z_{0}, 0, \ldots, 0\right)^{T}$, $\bar{z}_{2}(t)=\dot{\bar{\theta}}(t) \geq 0, \bar{\theta}(t) \in \mathcal{C}^{\hat{k}+1}$ and

$$
\forall t \geq 0:\left(\Phi_{1, \mathcal{P}}(\bar{z}(t)), \Phi_{2, \mathcal{P}}(\bar{z}(t))\right)^{T} \in \mathcal{N} \subset \operatorname{int}(\mathcal{X} \times \mathcal{U})
$$

Among these trajectories we can find solutions which are such that $\exists T \in$ $[0, \infty): \bar{z}(T)=\left(\theta_{T}, 0, \ldots, 0\right)^{T}$. Consequently, $\bar{v}(t)=\frac{d \bar{z}_{k}(t)}{d t}$ is an admissible input to OCP (18). Thus we have established feasibility of OCP (18) and the existence of a finite transition time $T$.

Step 2: The existence of an optimal solution to (18) can be deduced from two properties [15, Thm. 4, p. 259]: The existence of at least one admissible solution to the problem and the convexity and compactness of the extended velocity set $\mathcal{W}:=\left\{v \in \mathcal{V} \mapsto(A z+B v, 1)^{T} \in \mathbb{R}^{\hat{k}+2}\right\}$ for all fixed $z$. It is easy 
to see that linearity of (18b) and convexity and compactness of $\mathcal{V} \subset \mathbb{R}$ imply compactness and convexity of $\mathcal{W}$.

In step 1 of the proof we have constructed an admissible input $\bar{v}(t)$ for (18). Hence we can conclude from the existence of an admissible solution to the existence of an optimal solution to problem (18).

\section{References}

[1] A.P. Aguiar, J.P. Hespanha, and P.V. Kokotovic. Path-following for nonminimum phase systems removes performance limitations. IEEE Trans. Automat. Contr., 50(2):234-239, 2005.

[2] A.E. Bryson and Y.-C. Ho. Applied Optimal Control. Ginn and Company, Waltham, Massachusetts, 1969.

[3] F. Debrouwere, W. Van Loock, G. Pipeleers, Q.T. Dinh, M. Diehl, J. De Schutter, and J. Swevers. Optimal robot path following for minimal time versus energy loss trade-off using sequential convex programming. In Proceedings of the 2013 IEEE International Conference on Mechatronics (ICM), pages 316-320. IEEE, 2013.

[4] S. Devasia, D. Cheng, and B. Paden. Nonlinear inversion-based output tracking. IEEE Trans. Autom. Contr., 41(7):930-942, 1996.

[5] T. Faulwasser. Optimization-based solutions to constrained trajectory-tracking and path-following problems. Shaker, Aachen, Germany, 2013.

[6] T. Faulwasser, V. Hagenmeyer, and R. Findeisen. Optimal exact path-following for constrained differentially flat systems. In Proc. of 18th IFAC World Congress, Milano, Italy, pages 9875-9880, 2011.

[7] M. Fliess, J. Lévine, P. Martin, and P. Rouchon. Flatness and defect of nonlinear systems: introductory theory and examples. Int. J. Contr., 61(6):13271361, 1995.

[8] K. Graichen, V. Hagenmeyer, and M. Zeitz. A new approach to inversion-based feedforward control design for nonlinear systems. Automatica, 41(12):20332041, 2005.

[9] M. Guay. An algorithm for orbital feedback linearization of single-input control affine systems. Systems \& control letters, 38(4):271-281, 1999.

[10] V. Hagenmeyer. Robust nonlinear tracking control based on differential flatness. Fortschr.-Ber. VDI Reihe 8 Nr. 978, VDI Verlag, Düsseldorf, 2003.

[11] V. Hagenmeyer and E. Delaleau. Exact feedforward linearization based on differential flatness. Int. J. Contr., 76(6):537-556, 2003. 
[12] B. Houska, H.J. Ferreau, and M. Diehl. ACADO toolkit - an open-source framework for automatic control and dynamic optimization. Optimal Control Applications and Methods, 32(3):298-312, 2011.

[13] L. Jaulin, M. Kieffer, O. Didrit, and E. Walter. Applied Interval Analysis: With Examples in Parameter and State Estimation, Robust Control and Robotics, volume 12. Springer-Verlag, London, UK, 2001.

[14] S.M. LaValle. Planning algorithms. Cambridge University Press, 2006.

[15] E.B. Lee and L. Markus. Foundations of optimal control theory. The SIAM series in applied mathematics. John Wiley \& Sons New York, London, Sydney, 1967.

[16] J. Lévine. Analysis and control of nonlinear systems: a flatness-based approach. Mathematical Engineering. Springer, Berlin, 2009.

[17] T. Lipp and S. Boyd. Minimum-time speed optimization over a fixed path. Preprint, 2013.

[18] P. Martin, R.M. Murray, and P. Rouchon. Flat systems. In Proc. of the 4th European Control Conf, pages 211-264, 1997.

[19] M. B. Milam, K. Mushambi, and R. M. Murray. A new computational approach to real-time trajectory generation for constrained mechanical systems. In Proc. 39th IEEE Conf. Decision and Control, volume 1, pages 845-851, 2000.

[20] J. Oldenburg and W. Marquardt. Flatness and higher order differential model representations in dynamic optimization. Comp. \& Chem. Eng., 26(3):385 400, 2002.

[21] N. Petit, M.B. Milam, and R.M. Murray. Inversion based constrained trajectory optimization. In Proc. of 5th IFAC symposium on nonlinear control systems (NOLCOS), 2001.

[22] W. Respondek. Orbital feedback linearization of single-input nonlinear control systems. In 4th IFAC Conference on nonlinear control systems (NOLCOS), Enschede, The Netherlands, volume 98, pages 499-504, 1998.

[23] R. Rothfuß, J. Rudolph, and M. Zeitz. Flatness based control of a nonlinear chemical reactor model. Automatica, 32:1433-1439, 1996.

[24] P. Rouchon, M. Fliess, J. Lévine, and P. Martin. Flatness, motion planning and trailer systems. In Proc. 34th IEEE Conf. on Decision and Control, Piscataway, USA, volume 3, pages 2700-2705, 1993.

[25] H.J. Sira-Ramírez and S.K. Agrawal. Differentially flat systems. Control Engineering Series. Marcel Dekker Inc, New York, 2004.

[26] F. Suryawan, J. De Doná, and M. Seron. Minimum-time trajectory generation for constrained linear systems using flatness and b-splines. Int. J. Contr., 84(9):1565-1585, 2011. 
[27] F. Suryawan, J. De Doná, and M. Seron. Splines and polynomial tools for flatness-based constrained motion planning. International Journal of Systems Science, 43(8):1396-1411, 2012.

[28] V.A. Topogonov. Differential Geometry of Curves and Surfaces - A Concise Guide. Birkhäuser, Boston, 2006.

[29] D. Verscheure, B. Demeulenaere, J. Swevers, J. De Schutter, and M. Diehl. Time-optimal path tracking for robots: A convex optimization approach. IEEE Trans. Autom. Contr., 54(10):2318-2327, 2009.

[30] U. Vollmer and J. Raisch. Control of batch cooling crystallization processes based on orbital flatness. Int. J. Contr., 76(16):1635-1643, 2003. 